\title{
An eye for inspiration
}

\author{
The discovery that the eye of a particular mantis shrimp has an achromatic quarter-waveplate that is \\ superior to modern-day devices could be a source of inspiration to those designing optical components. \\ Nature Photonics spoke to Nicholas Roberts, one of the researchers involved in the study.
}

Tell us a little about these mantis shrimps.

The types of mantis shrimps we investigated in our study are found in and around coral reefs in the Indo-Pacific area, but there are other species that inhabit temperate waters and the deep ocean. It is thought that their ancestors came into existence 400 million years ago. Some live in burrows that they dig, whereas others inhabit holes in the reef, defending them with a punch that can break glass and shatter bone.

\section{Why did you decide to study them?} Research into these animals has been driven largely by their unusual eyes, and originally dates back to the 1890 s with a scientist called Exner. Our team has been working since the early 1980s to understand how these remarkable eyes work. Not only do they have 12-channel colour vision, but they can also distinguish between different forms of circularly and linearly polarized light. From a biological perspective, it really is the most complex animal visual system yet discovered.

\section{Why do these creatures have such sophisticated vision?}

That's a good question, and there are several possibilities. One is that they need a highly developed visual system for finding and catching food. They are predators that strike very rapidly - so fast that they can cause cavitation in the water. As for polarization sensitivity, at the moment this seems linked with sexual signalling and communication because males and females show different circularly polarized reflections. As other animals do not seem to possess this sort of vision, it may be for secret or covert communication that does not attract the attention of others. Alternative possibilities include the use of complex polarization vision for object detection and for improving image clarity in water. Something we would like to make clear though is that this latest discovery isn't really about the circularly polarized vision of these creatures, as that's well-known and has already been reported. Instead, what we have done now is revealed the unique optical design and mechanism of

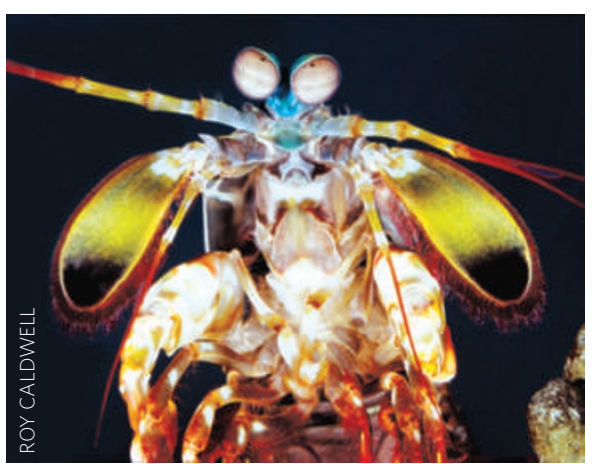

The mantis shrimp (Odontodactylus scyllarus) can see different forms of circularly polarized light, an ability enhanced by a quarter-waveplate that outperforms synthetic designs.

the achromatic quarter-waveplate - which makes it possible to change one form of polarization into another - within its eye. The optical design has exceptional performance; it is one that humans were previously unaware of and hasn't yet been exploited.

\section{Were you surprised by the level of achromaticity in the mantis shrimp's quarter-waveplate?}

We were completely surprised. In the lab I'm very used to working with commercial zero-order quarter-waveplates in optics experiments, and you don't have to change the wavelength much before they cease to work well. But to see results with this level of achromaticity when compared with synthetic designs was amazing. Scientists have known for a long time that forms of birefringence can be found in biological samples - particularly in photoreceptor cells. But here, it is used for a specific purpose, and from an optics perspective the really exciting thing about this research is the simplicity of the mechanism; if you roll some cell membranes into tubes with the correct properties and dimensions, you get an amazingly achromatic device - it is beautifully simple. Furthermore, it's all achieved with a biological system that self-assembles and has a low refractive index difference between the lipids in the membranes and the surrounding cytoplasm. Compared with synthetic designs, it is elegant, simple and performs astonishingly well. The need for this rather exquisite quarter-wave tuning in the eye is not clear. However, it improves the polarization contrast of their vision throughout the whole visible spectral range. This mechanism exists perhaps because it provides some evolutionary advantage - it only requires small modifications to the existing photoreceptor architecture.

\section{Could the design be transferred to} man-made devices?

I certainly believe this may be possible. We know that lyotropic hexagonal liquid-crystal phases have a similar microtubule structure with amphiphillic molecules. If you can chemically engineer the correct optical properties, and if the device is of the correct optical thickness, then there is no reason that the same optical principles won't apply. Liquid crystals are also easily polymerizable, so there is even a route to lock-in these optical properties and make a stable thinfilm device. We can take great inspiration from nature; one of the best examples of exploiting biological eye designs is from lobsters, which have reflecting-type compound eyes. This design was the recent inspiration for a wide-angle X-ray detector used in an astronomical telescope.

\section{What are your future plans for work in this area?}

Some of our other work being done right now suggests that different species have a range of circular polarization sensitivities, ranging from nearly insensitive through to $100 \%$ sensitive, so we're looking at the interspecies variation in the optical design of the cell type and their dimensions to understand why that is. Also, from a modelling perspective, we're currently looking at the angular dependence of this mechanism and working towards a full three-dimensional optical model.

\section{INTERVIEW BY OLIVER GRAYDON}

Nicholas Roberts and his co-workers have a Letter on biological achromatic waveplates on page 641 of this issue. 